\title{
Monte Carlo simulation of low temperature phase diagrams of $\mathrm{YBa}_{2} \mathrm{Cu}_{3} \mathrm{O}_{6+x}$
}

\author{
Rita Khanna ${ }^{\text {a }}$, T.R. Welberry ${ }^{a}$ and G. Ananthakrishna ${ }^{b}$ \\ a Research School of Chemistry, The Australian National University, PO Box 4, Canberra, ACT 2601, Australia \\ b Materials Research Centre, Indian Institute of Science, Bangalore 560012, India
}

Received 30 October 1991

Revised manuscript received 13 February 1992

\begin{abstract}
We report the results of Monte Carlo simulation of oxygen ordering in the oxygen deficient portion $(x<0.5)$ of $\mathrm{YBa}_{2} \mathrm{Cu}_{3} \mathrm{O}_{6+x}$ at low temperatures. We find qualitative agreement among cluster - variation, Monte Carlo and transfer matrix methods. However, low temperature and ground state simulations clearly indicate the presence of a tetragonal phase. There is also evidence for two second order phase transition lines separating the tetragonal and the "double cell" ortho II phase. The effect of decreasing the inter-chain repulsion on oxygen ordering has also been investigated.
\end{abstract}

\section{Introduction}

The structural phase transitions of the high temperature superconductor $\mathrm{YBa}_{2} \mathrm{Cu}_{3} \mathrm{O}_{6+x}$ have been intensively investigated both from the theoretical and experimental points of view. It is now well established that the superconducting properties of $\mathrm{YBa}_{2} \mathrm{Cu}_{3} \mathrm{O}_{6+x}$ are very sensitive to the oxygen stoichiometry and to the oxygen ordering in the $\mathrm{Cu}-\mathrm{O}$ basal plane [1-10]. It is therefore very important to have a clear understanding of the phase diagram of $\mathrm{YBa}_{2} \mathrm{Cu}_{3} \mathrm{O}_{6+x}$. Theoretically, apart from the work of Khachaturyan and Morris [11], most treatments reduce the problem to a two-dimensional ordering of the oxygen atoms in the basal plane. The basal plane may be described as a square lattice of $\mathrm{Cu}$ ions with the oxygen sites between the adjacent $\mathrm{Cu}$ ions. The oxygen sites may be vacant or occupied depending on the magnitude of $x$. In order to explain ordering in this plane, a lattice gas (2D Ising) model was proposed by de Fontaine et al. [12], assuming short range effective pair interactions between oxygen sites. An occupied site may be represented by spin $S_{i}=+1$ (up), an empty site by $S_{i}=-1$ (down). The Hamiltonian is equivalent to an Ising model in a magnetic field with three effective pair interactions $J_{1}, J_{2}$ and $J_{3}$ :

$$
H=J_{1} \sum_{\mathrm{nn}} S_{i} S_{j}+J_{2} \sum_{\mathrm{nnn}} S_{i} S_{j}+J_{3} \sum_{\mathrm{nnn}}^{\prime \prime} S_{i} S_{j}-H \sum S_{i}
$$

The sum nn extends over all nearest neighbour interactions. The sums nnn extend over next nearest neighbour (nnn) bonds, where the prime indicates $\mathrm{nnn}$ bonds mediated by a $\mathrm{Cu}$ ion and the double primes without the $\mathrm{Cu}$ ion. The strength of the interaction parameters, confined to the range $[-1,1]$, completely determines the energy of the oxygen-ion configurations and the stability of various ordered phases. There is enough experimental evidence for the existence of a tetragonal phase $(x \sim 0)$, the orthorhombic phase $(x \sim 1)$ and "double cell" orthorhombic phase $(x \sim 0.5)[1-10]$. There are also reports of "magneli phases" $(x>0.5)$ but these appear to be metastable in nature [13]. Apart from some recent work using ab initio calculations, most investigators have used $J_{1}=1, J_{2}=-0.5$ and $J_{3}=0.5$, as originally used by Wille et al. [14,15]. This choice of parameters has been traditionally used despite the fact that $J_{3}$ is only weakly repulsive. The interactions $J_{1}$ and $J_{2}$, being of chemical origin, must be appreciably stronger than the purely coulombic interchain repulsion $J_{3}$ [ 16 ]. In addition, a proper choice of the sign and magnitude of $J_{3}$ is crucial to the stability of 
the ortho II phase. A positive $J_{3}$ will result in the complete disappearance of the ortho II phase.

There appears to be a slight controversy about the existence of a tetragonal phase at low temperatures in the oxygen deficient region $(x \leq 0.5)$ of the phase diagram. Monte Carlo simulations of Aukrust et al. [17] indicate a broad tetragonal region extending from $x=0.0$ to $x=0.24$. These computations, however, are restricted to slightly higher temperatures and the low temperature results are obtained through extrapolation. Kikuchi and Choi [18] using the cluster variation method, indicate complete absence of a tetragonal phase at low temperatures and show the existence of a new phase labelled as $\overline{\mathrm{OI}}$. Using a different set of parameters $\left(J_{1}=1.0, \quad J_{2}=-0.75\right.$, $\left.J_{3}=0.5\right)$ and Monte Carlo simulation, we observed a much narrower tetragonal phase [19]. In order to obtain a clear picture of the phase diagram at low temperatures, we investigate, in this paper, the stability of different phases and their dependence on the strength of the $J_{3}$ interaction parameter. Using Monte Carlo simulation, we investigate the low temperature region of the oxygen deficient $(x \leq 0.5)$ portion of the phase diagram. Computer programs were especially designed to overcome the problem of underflow often encountered in low temperature simulation.

\section{Monte Carlo simulations}

In our Monte Carlo simulations, we considered a system of $N=2 \times L \times L$ spins with periodic boundary conditions, $L$ being measured in units of the lattice constant $a$. The simulations were performed using single spin-flip Glauber dynamics in the grand canonical ensemble, with the oxygen concentration varying as a function of temperature and magnetic field. Starting from an initial configuration, the system was allowed to evolve according to the following algorithm: using pseudorandom numbers one generates a change of configuration $X \rightarrow X^{\prime}$. This transition $\mathrm{X} \rightarrow \mathrm{X}^{\prime}$ is taken to be the flip $S_{i} \rightarrow-S_{i}$ of a randomly chosen spin. The energy change $\delta U=H\left(\mathrm{X}^{\prime}\right)-H(\mathrm{X})$ is then computed. The transition probability

$$
W=\exp \left(-\delta U / k_{\mathrm{B}} T\right) /\left[1+\exp \left(-\delta U / k_{\mathrm{B}} T\right)\right]
$$

is then compared with a random number $\eta$, chosen uniformly in the range [0,1]. If $W>\eta$ the transition is performed. If $W<\eta$ the attempted change $\mathrm{X}^{\prime}$ is rejected and $\mathrm{X}$ is counted once more for averaging. For $\mathrm{T}=0.0$ simulations, a transition was accepted only if it lowered the energy $U$ of the system and was rejected otherwise. The simulations were carried out for lattice sizes in the range $20 \leq L \leq 96$. The data was obtained for typically ten to twenty thousand Monte Carlo steps per site.

Since the ortho I, ortho II and tetragonal phases are predicted by the present model, it is appropriate to define the sublattice magnetisations (see fig. 1) and the order parameters corresponding to the different phases. We define the two order parameters for the ortho I and ortho II phases respectively as

$$
\begin{aligned}
M_{\mathrm{I}}= & \left\{\sum_{i=1}^{4} m_{i}-\sum_{i=5}^{8} m_{i}\right\} / 8, \\
M_{\mathrm{II}}= & \left\{\left(m_{1}+m_{2}\right)-\left(m_{3}+m_{4}\right)\right. \\
& \left.\quad+\left(m_{5}+m_{6}\right)-\left(m_{7}+m_{8}\right)\right\} / 4,
\end{aligned}
$$

where the eight sublattice magnetisations are defined by

$$
m_{\alpha}=(8 / N)\left\langle\sum_{i \in \alpha} S_{i}\right\rangle, \quad \alpha=1,2, \ldots, 8
$$

It is clear that $M_{\mathrm{I}}$ is unity in the ortho I phase, zero in the tetragonal phase and takes on a value of 0.5 in the ortho II phase. Similarly $M_{\mathrm{II}}$ takes on a value of unity in the ortho II phase and goes to zero in the other two phases. The total magnetisation is

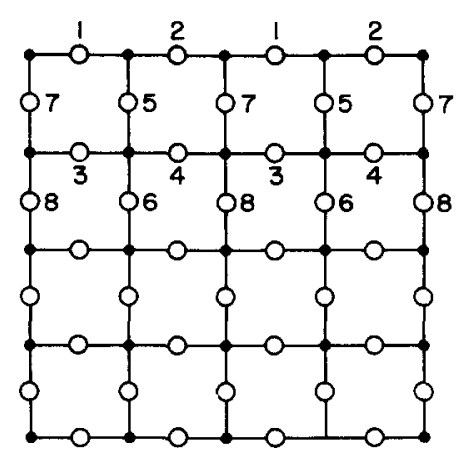

Fig. 1. $\mathrm{Cu}-\mathrm{O}$ basal plane showing the eight sublattices. 
$M=(1 / N) \sum_{i}\left\langle S_{i}\right\rangle$

The concentration $x$ of oxygen can be represented in terms of the magnetisation $M$ as: $x=(1+M) / 2$. Order parameter distribution functions were used to locate the phase boundary and to determine the order of the transition [20]. $P(\phi) \mathrm{d} \phi$ is defined as the probability the order parameter will take on a value in the range $[\phi, \phi+\mathrm{d} \phi]$. The $P(x)$, the ortho $\mathrm{I}$ and ortho II order parameters were used in this analysis. Throughout the transition region $P(\phi)$ is a double peaked function. At the transition temperature, the two peaks have the same intensity. For a second order transition, it is an important requirement that the two peaks move closer with increasing system size. An opposite size dependence in $P(\phi)$ indicates a first order phase transition. $P(\phi)$ is therefore indispensible for determining the nature of the transition.

\section{Simulations at $\boldsymbol{T}=0.0$}

In zero temperature simulations, it is quite possible that the system may get locked in a metastable state and the results may not be a true representation of the equilibrium state. The simulations were repeated for different sets of initial conditions, random number generators and lattice sizes. Similar results were obtained in all cases, thereby gaining in credibility as good representations of equilibrium ground states.

$x$ versus $H /\left|J_{1}\right|$ plots for $J_{3}=0.5$ to $0.3\left(J_{1}=1.0\right.$, $\left.J_{2}=-0.5\right)$ are shown in figs. $2(\mathrm{a}-\mathrm{c})$. The results are very interesting. For $J_{3}=0.5$, there is clear evidence for three stable states, i.e., T, OII and OI. This result agrees well with other predictions of ground states for this set of interaction parameters [21]. It is worth pointing out that there was hardly any change in fig. 2 after 1000 MCS. The system, when away from the transition region, relaxed very quickly to the stable configuration.

With decreasing magnitude of $J_{3}$ (figs. 2(a-c)), the ortho II region became narrower and narrower, finally disappearing completely at $J_{3}=0.2$. This result is hardly surprising as the stability of ortho II phase is governed by the strength of $J_{3}$ relative to that

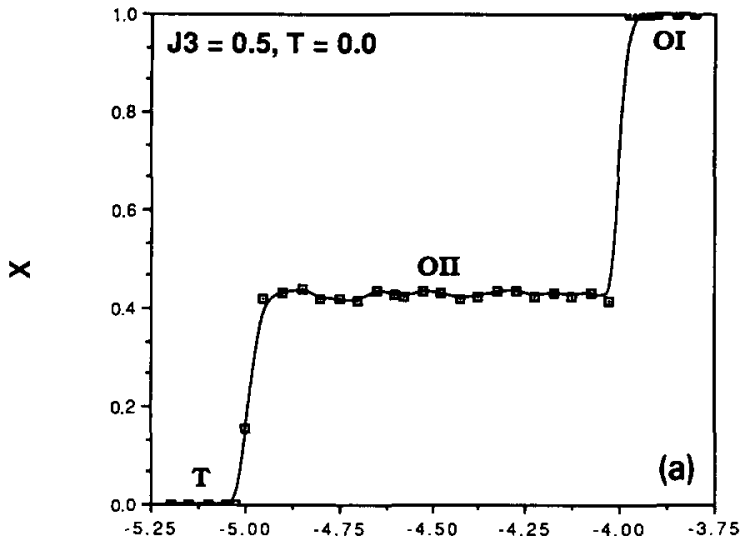

H

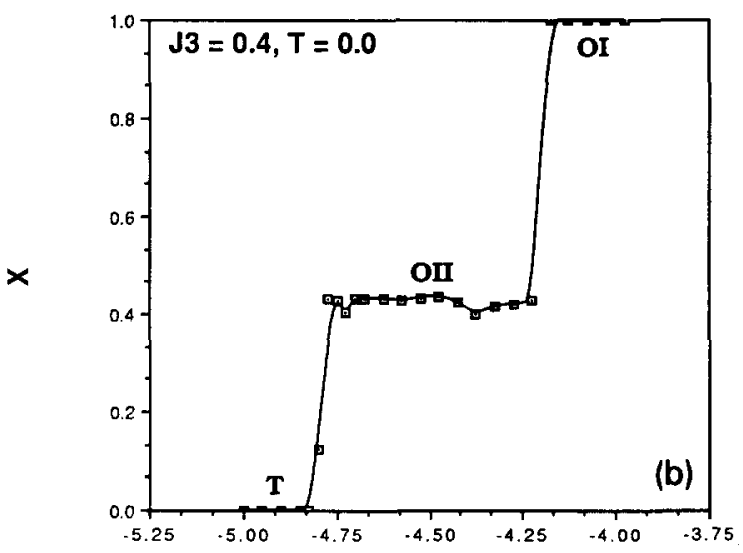

H

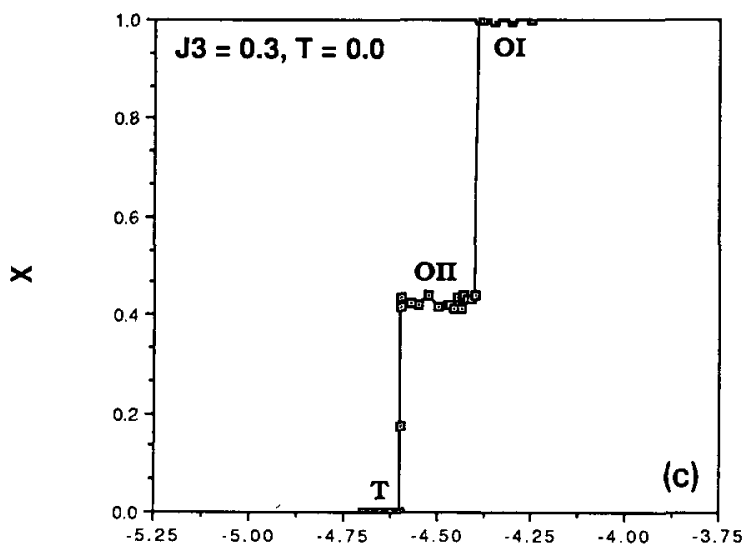

H

Fig. 2. Ground state simulation plots of oxygen concentration $x$ vs. magnetic field $H /\left|J_{1}\right|$ for various values of the $J_{3}$ parameter. 
of $J_{2}$. Only the T and OI phases are stable with an attractive $J_{3}$ [11]. According to our simulation results, it now appears that the OII phase is not a stable ground state even for slightly positive values of $J_{3}$. We did not find any evidence for magneli phases as stable ground states in these simulations.

\section{Simulations of finite temperatures}

Our phase diagrams obtained from the analysis of distribution function data from the Monte Carlo simulations are shown in figs. $3(\mathrm{a}-\mathrm{c})$ for $J_{3}$ in the range 0.3 to 0.5 . In this work, we have restricted our attention to the oxygen deficient low temperature region of the phase diagram with $x$ ranging from 0 to 0.5 . The dimensionless temperature scale $k_{\mathrm{B}} T /\left|J_{1}\right|$ in these results is four times the scale used by Kikuchi and Choi and Aukrust et al. (this is due to the fact that in eq. ( 1 ) we have used the variable $S_{i}$ having a magnitude $+1(-1)$ to represent an occupied (empty) site. Aukrust et al. on the other hand use the variable $C_{i}$ which has a magnitude $+1(0)$ for an occupied (empty) site. Transformation of variables using $S_{i}=\left(2 C_{i}-1\right)$ yields $J_{1}=\phi_{\mathrm{NN}} / 4$, resulting in our temperature scale to be four times larger). In all three phase diagrams there is clear evidence for two phase boundaries separating the tetragonal and ortho II phase at low temperatures. The two boundaries come closer with increasing temperature, finally merging into a single phase boundary. The intermediate phase has been identified as the low density, low temperature orthorhombic phase $\overline{\mathrm{OI}}$ that has been proposed by other authors [18,22-25].

Figures 4 and 5 show plots of the distribution function $P(x)$ versus $x$ for $J_{3}=0.3$ at $k_{\mathrm{B}} T /\left|J_{1}\right|=0.5$ across the ortho II and $\overline{\mathrm{OI}}$ phase boundary and across the $\overline{\mathrm{OI}}$ and $\mathrm{T}$ phase boundary, respectively. In these plots, $P(x)$ has not been normalised and the plots are typically for $10000 \mathrm{MCS}$. Away from the transition region, $P(x)$ is a single peaked function and is quite sharp. A much broader/double peak region in the distribution function implies that more than one state is being populated, thereby indicating the transition region. After approximately locating the transition region with a small lattice size $(32 \times 32)$, we carried out simulations with increasing lattice size, i.e., $48 \times 48,64 \times 64$ etc. Figure 4 (a) shows the re-
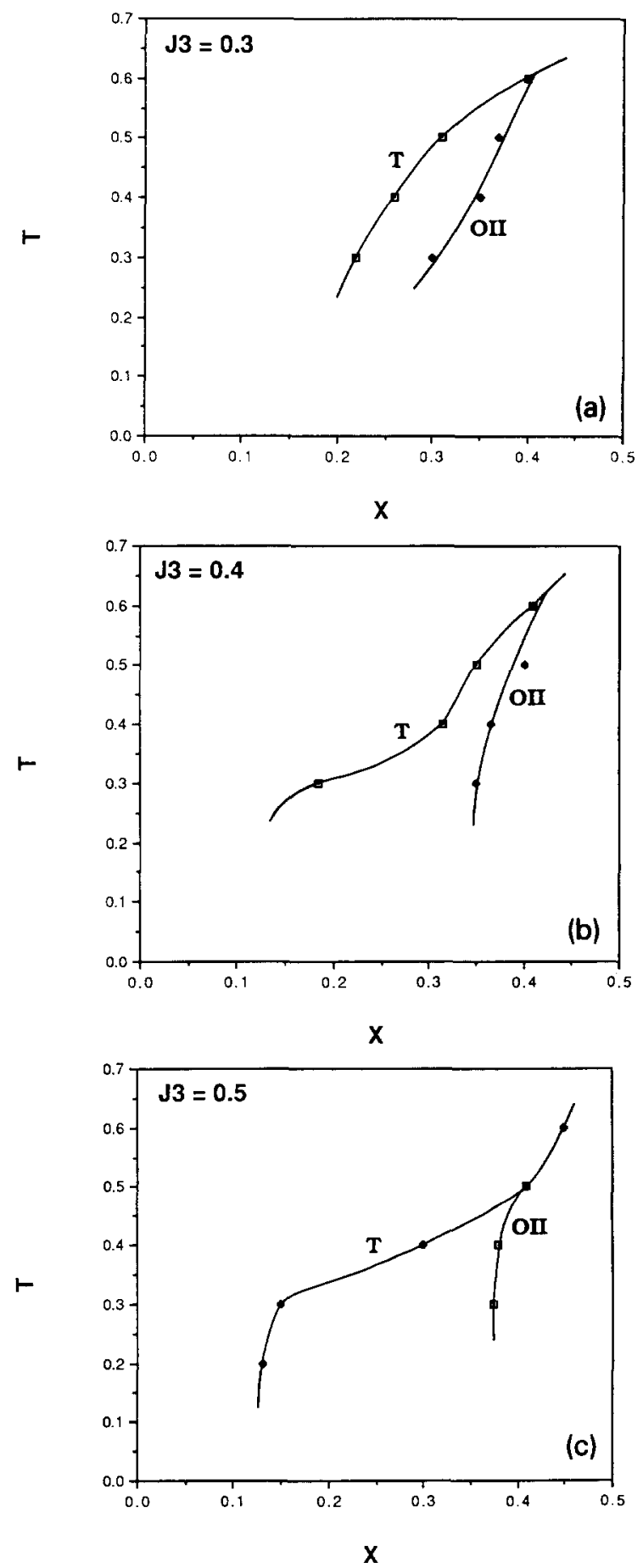

Fig. 3. Low temperature phase diagrams of $\mathrm{YBa}_{2} \mathrm{Cu}_{3} \mathrm{O}_{6+x}$ as obtained from the analysis of distribution function data for various values of the $J_{3}$ parameter. 

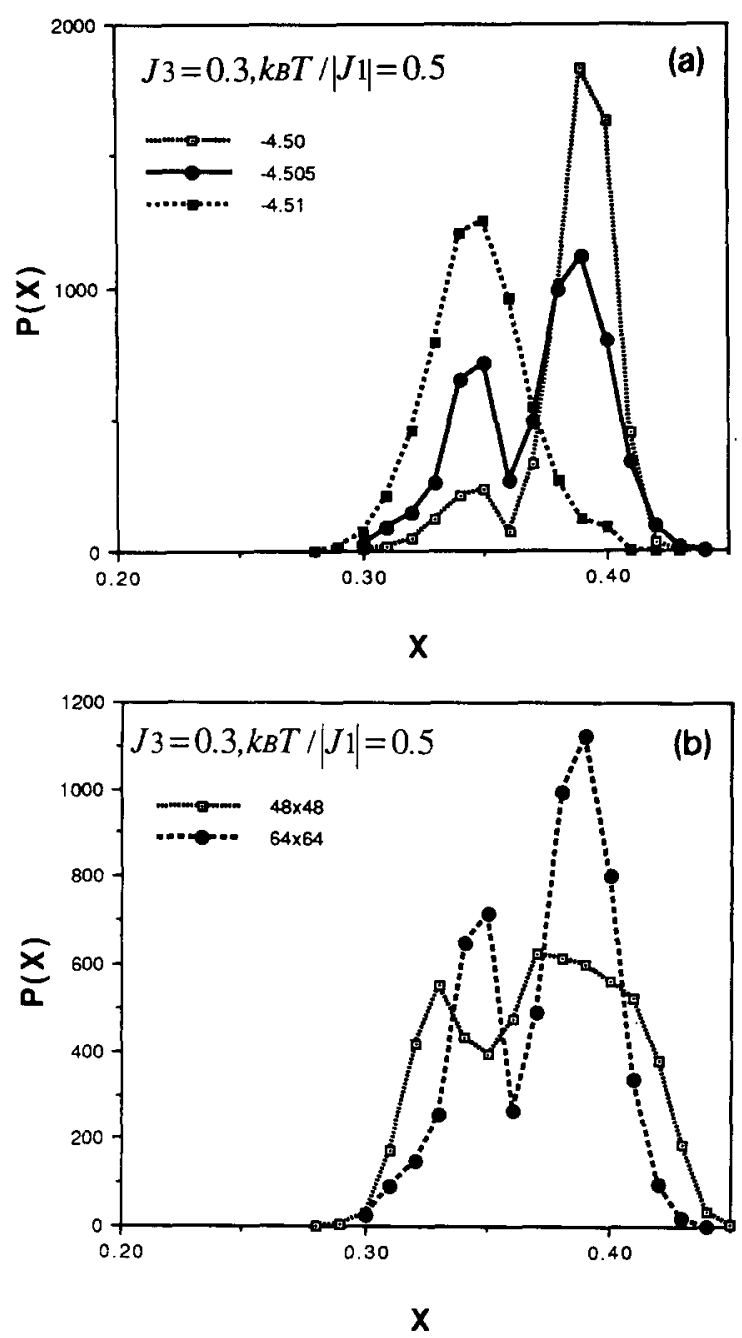

Fig. 4. Plots of distribution function $P(x)$ vs. $x$ for $J_{3}=0.3$ and temperature $k_{\mathrm{B}} T /\left|J_{1}\right|=0.5$ for ortho II to $\overline{\mathrm{Ol}}$ transition. (a) Plots of $P(x)$ for three different values of the field $H /\left|J_{1}\right|$. The magnitude of $H /\left|J_{1}\right|$ is indicated against the plot symbol. The lattice size for these simulations was $64 \times 64$. (b) Plots of $P(x)$ for two different lattice sizes at $H /\left|J_{1}\right|=-4.505$.

sults for a $64 \times 64$ lattice for different field values across the OII to $\overline{\text { OI }}$ transition and fig. 5(a) shows the corresponding plots for the $\overline{\mathrm{OI}}$ to $\mathrm{T}$ transition region for a $32 \times 32$ lattice. Figures $4(\mathrm{~b})$ and 5 (b) show the lattice size dependence of $P(x)$ in the transition region for the two phase boundaries. Even though the double peak region persists with increasing lattice size, the peaks become increasingly sharper and move closer. This size dependence feature clearly in-
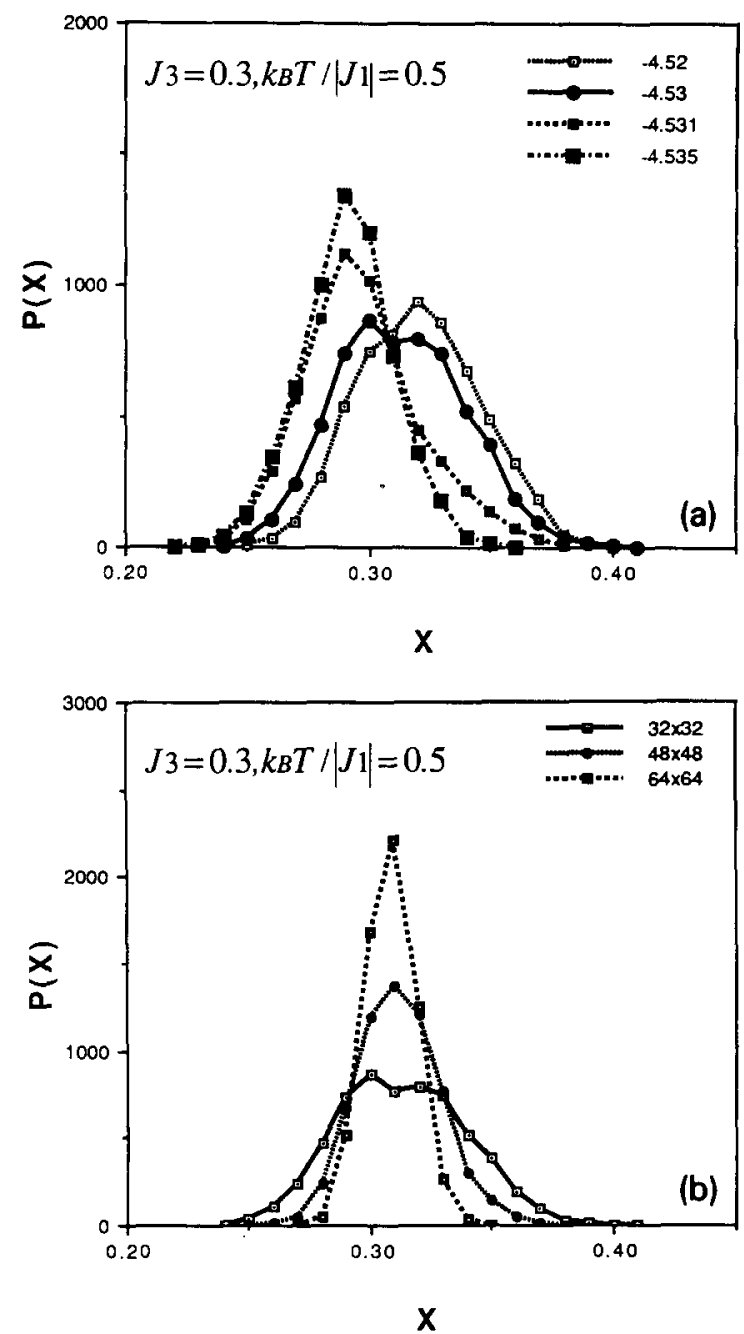

Fig. 5. Plots of the distribution function $P(x)$ vs. $x$ for $J_{3}=0.3$ and temperature $k_{\mathrm{b}} T /\left|J_{1}\right|=0.5$ for the $\overline{\mathrm{OI}}$ to $\mathrm{T}$ transition. (a) Plots of $P(x)$ for three different values of the field $H /\left|J_{1}\right|$. The magnitude of $H /\left|J_{1}\right|$ is indicated against the plot symbol. The lattice size for these simulations was $32 \times 32$. (b) Plots of $P(x)$ for two different lattice sizes at $H /\left|J_{1}\right|=-4.53$.

dicates that both the transitions are of second order. Similar results were obtained for $J_{3}=0.4$ and $J_{3}=0.5$. In our earlier work [19] using critical slowing down and the relaxation time, $\tau$, to locate a phase transition, it is possible to miss two such close lying transitions and to interpret them as a single transition. However, the distribution function approach using a number of order parameters has a better resolution 
and two close-lying transitions can be unambiguously distinguished.

Apart from the twin phase boundaries, the extent of the tetragonal region at low temperatures appears to depend on the strength of $J_{3}$ for a given set of $J_{1}$ and $J_{2}$ values. A smaller magnitude of $J_{3}$ relative to that of $J_{2}$ appears to stabilise the tetragonal phase at low temperatures, whereas a bigger $J_{3}$ appears to strengthen the ortho II phase and shrinks the $\mathrm{T}$ phase closer to $x=0.0$.

\section{Discussion and concluding remarks}

Qualitatively the phase boundaries computed in this work are consistent with those obtained by Wille et al., Kikuchi and Choi and Aukrust et al. Kikuchi and Choi, in their CVM simulations have indicated two lines of phase transitions separating $\mathrm{T}$ and OII phase, but they predict the transition to be of first order. Our results very clearly indicate that they are of second order. This is in complete agreement with the detailed scaling analysis of Monte Carlo and transfer matrix data by Aukrust et al. The phase transition line adjacent to the OII phase in our $J_{3}=0.5$ simulation, matches exactly with the corresponding line in the phase diagram simulated by Aukrust et al., but apparently they have missed the second transition line adjacent to the $T$ phase, presumably because their data were restricted to slightly higher temperatures. Secondly, Kikuchi and Choi predict a complete absence of the $T$ phase at low temperatures. Out $T=0.0$ and low temperature data indicate the presence of the $T$ phase, albeit a much narrower one than the one predicted by the extrapolation of the data of Aukrust et al.

For $J_{3}=0.5$, oxygen chains tended to span the entire lattice. However, in the $T$ region, chains were of much smaller size and were seen to run along both directions. Similarly with the decreasing magnitude of $J_{3}$, the chain length became much smaller than the lattice size. In addition, hysteresis effects were noticed for very low temperature runs. For these temperatures ( $T \leq 0.3)$, simulations were carried out on bigger lattice sizes ( $L=80,96$, etc) and for typically twenty to thirty thousand MCS. Location of the T phase boundary and the order of the transition was quite unambiguous. Recently Rikvold et al. [26] have looked at the macroscopic effects of local oxygen fluctuations in $\mathrm{YBa}_{2} \mathrm{Cu}_{3} \mathrm{O}_{6+x}$. Using $J_{3}=0.5$ and a $32 \times 32$ lattice in their Monte Carlo simulation, they did not find evidence for the $\overline{\text { OI }}$ phase and their lattice appeared to consist of short chains of oxygen atoms, randomly dispersed and oriented. This result is contrary to our results and that of many other authors $[18,22-25]$. We observe very clear evidence for two phase boundaries in the low temperature, oxygen deficient region of the phase diagram. We found it essential to increase the lattice size and number of Monte Carlo steps per site for low temperature simulations to avoid hysteresis effects.

In this paper we have attemped to use more realistic values of the interaction parameters and have investigated how these small changes may affect the stability of different states and the associated phase diagrams. We find that Monte Carlo simulation using the distribution function approach is well suited to computing phase diagrams. These simulations are still of a preliminary nature and for comparing simulation results with the experimental data, a more realistic model of oxygen ordering is required. Such a model should include the effect of longer range interactions, better potentials, the effect of lattice relaxations, etc. We are currently investigating the effect of lattice relaxation on oxygen ordering.

\section{References}

[1] G. Van Tendeloo, H.W. Zandbergen and S. Amelinckx, Solid State Commun. 63 (1987) 603.

[2] H.W. Zandbergen, G. van Tendeloo, T. Okabe and S. Amelinckx, Phys. Status Solidi A 103 (1987) 45.

[3] R.J. Cava, B. Batlogg, C.H. Chen, E.A. Rietman, S.M. Zahurak and D. Weber, Phys. Rev. B 36 (1987) 5719.

[4] J.D. Jorgenson, M.A. Beno, D.G. Hinks, L. Soderholm, K.J. Volin, R.J. Hitterman, J.D. Grace, I.K. Schuller, C.V. Segre, K. Zhang and M.S. Kleefisch, Phys. Rev. B 36 (1987) 3608.

[5] F. Beech, S. Miragalia, A. Santoro and R.S. Roth, Phys. Rev. B 35 ( 1987) 3608 .

[6] C.H. Chen, D.J. Werder, L.F. Schneemeyer, P.K. Gallagher and J.V. Waszczak, Phys. Rev. B 38 (1988) 2888.

[7] E.D. Specht, C.J. Sparks, A.G. Dhere, J. Brynestad, O.B. Cavin, D.M. Kroeger and H.A. Oye, Phys. Rev. B 37 (1988) 7426.

[8] R. Beyers, B.T. Ahn, G. Gorman, V.Y. Lee, S.S.P. Parkin, M.L. Ramirez, K.P. Roche, J.E. Vanquez, T.J. Gur and R.A. Huggins, Nature 340 (1989) 619. 
[9] R. Beyers and T. Shaw, in: Solid State Physics eds. H. Ehrenrich and D. Tuenbull, Vol. 42 (Academic, New York, 1989) p. 135.

[10] J. Rayes-Gasga, T. Krekel, G. van Tendeloo, J. van Landyut, S. Amelinckx, W.H.M. Bruggink and M. Verwij, Physica C 159 (1989) 831.

[11] A.G. Khachaturyan and J.W. Morris Jr., Phys. Rev. Lett. 59 (1987) 2776; ibid., 61 (1988) 215.

[12] D. de Fontaine, L.T. Wille and S.C. Moss, Phys. Rev. B 36 (1987) 5709.

[13] V.E. Zubkus, S. Lapinskas and E.E. Tornau, Physica C 159 (1989) 501.

[14] T. Wille and D. de Fontaine, Phys. Rev. B 37 (1988) 2227.

[15] A. Berera, L.T. Wille and D. de Fontaine, J. Stat. Phys. 50 (1989) 1245.

[16] L.G. Mamsurova, K.S. Pigalskiy, V.P. Sakun, A.I. Shushin and L.G. Scherbakova, Physica C 167 (1990) 11.

[17] T. Aukrust, M.A. Novotny, P.A. Rikvold and D.P. Landau, Phys. Rev. B 41 (1990) 8772.
[18] R. Kikuchi and J.S. Choi, Physica C 160 (1989) 347.

[19] R. Khanna and G. Ananthakrishna, Physica C 195 (1992) 59

[20] O.G. Mouritsen, in: Computer Studies of Phase Transitions and Critical Phenomenon (Springer, New York, 1984), p. 18 .

[21 ] J. Stolze, Phys. Rev. Lett. 64 (1990) 970.

[22] D. de Fontaine, M.E. Mann and G. Ceder, Phys. Rev. Lett. 63 (1989) 1300.

[23] N.C. Bartlet, T.L. Einstein and L.T. Wille, Phys, Rev. B 40 (1989) 10759.

[24] D. de Fontaine, G. Ceder and M. Asta, Nature 343 (1990) 544.

[25] G. Ceder, M. Asta, W.C. Carter, M. Kraitchman, D. de Fontaine, M.E. Mann and M. Sluiter, Phys. Rev, B 41 (1990) 8698.

[26] P.A. Rikvold, M.A. Novotny and T. Aukrust, Phys. Rev. B 43 (1991) 202 